\title{
Design of a lab component on switching mode DC-DC converters for analog electronics courses
}

\section{Dr. Yoon G. Kim, Calvin College}

Yoon G. Kim received his M.S. and D.Sc. degrees in Electrical Engineering from Washington University in St. Louis in 2000 and 2005, respectively. He is a faculty member of the Electrical and Computer Engineering program in the Engineering Department at Calvin College in Michigan and teaches courses in analog electronics and control systems. He has over 11 years of industrial Research and Development experience in the area of telecommunication systems, where he designed analog and digital systems, including digital set-top-box converters for Hybrid Fiber Coax cable networks, digital video scramblers, data acquisition systems, analog subscriber line interface systems, DC-DC converters, DC-AC converters, and microprocessor boards. His research interests include Wireless Sensor Networks, Power Electronics and Embedded Systems. 


\title{
Design of a lab component on switching mode DC-DC converters for analog electronics courses
}

\begin{abstract}
This paper presents a practical approach to teaching an introductory-level laboratory experiment focused on the analysis of switching-mode DC-DC converters. This experiment would take place in an analog electronics course as a laboratory component for junior undergraduate electrical and computer engineering students.

Switching mode power supplies have been used in industries and consumer electronics for many decades. Recently, they have become pervasive in our daily life as many smartphones, portable computing devices, and renewable energy systems are using switching mode power converters. Due to the wide acceptance of this technology, a lab component has been added in an analog electronics course to introduce switching-mode DC-DC conversion.
\end{abstract}

The approach taken is to integrate students' knowledge and experiences with electronic discrete components such as BJTs, diodes, inductors and other passive devices and to enhance their learning about non-linear circuits utilizing low-voltage, low-power switching power converter experiments. The lab is designed to use general purpose components, which are usually available in electrical engineering laboratories and is designed to avoid special-purpose switching power conversion ICs, which embed all of the function blocks necessary (including switching transistors) inside the ICs. These ICs are specially designed to reduce the overall manufacturing cost of switching power supplies. Although using these ICs is a cost effective way to massproduce power supplies, using them in an undergraduate-level electronics lab would limit the students' learning about the theory behind switching power supplies. The experiments in the lab utilize BJTs, Schottky-barrier diodes, inductors, and oscillator circuits running at $100 \mathrm{kHz}$ as the switching frequency. The experiment starts by exploring a boost type converter topology, demonstrating to students that such circuits can produce a voltage higher than the input voltage. This should arouse the student's curiosity and motivate them to get engaged in the further lab experiments.

This paper includes complete working schematics, simulation results, measurement data, part numbers, and vendor lists. It would enable any instructor to easily adopt the DC-DC converter lab in their analog circuit courses.

After all experiments have been completed, the students submit a lab report which includes objectives, results/discussion, and conclusions. All sixteen junior students have successfully completed the lab. The conclusions of the reports and the survey results have shown that they found the lab beneficial in their understanding of switching-mode DC-DC Converters. 


\section{Introduction}

A modern switching mode DC-DC converter is a power supply, which consists of semiconductor switching devices (e.g., transistors and diodes) and energy storage elements, such as inductors and capacitors. In general, the DC-DC converters are used to convert the voltage level of the input into a different voltage level of the output. The switching mode power supplies have been used for many decades, as the technology enables a compact and light-weighted design and provides higher electrical energy conversion efficiency. One of the great applications was the Apollo spacecraft, which used DC-DC converters with a switching frequency of $1.6 \mathrm{kHz}$.

The DC-DC converters have been widely accepted recently, especially due to the growing prevalence of the use of portable electronics, such as smartphones and tablet computers, where the batteries are the primary energy sources, and the increased acceptance of solar energy systems, in which the converters are used to convert the energy harvested from photovoltaic cells, whose voltage is one level of DC, to another level of DC for charging batteries.

Due to the widely accepted technology, many higher educations have been including power electronics courses in their undergraduate curriculum ${ }^{2,3,5,6}$ and their graduate programs ${ }^{4}$.

In this paper, a practical approach to teaching an introductory-level lab experiment on DC-DC converters is presented. This lab experiment has been included in a junior level analog electronics course as a lab component. The target students were junior electrical and computer engineering students who had completed a course on op amp circuits and electronic devices, such as BJTs, diodes, inductors, and op amps.

The approach is to integrate students' prior knowledge and experiences with electronic devices; and to introduce DC-DC converters using a lab experiment. The lab is aimed at experimenting with DC-DC converters as a dual power supply, which provides positive and negative voltages for an inverting op amp circuit, which students are familiar with. The converter circuits take a single $+5 \mathrm{~V}$ source and generate $\pm 15 \mathrm{~V}$. Students could easily test the converter circuits by checking the amplifier output signal. The students begin with an oscillator circuit and add components, in a step by step fashion, until they complete the dual power supply and op amp amplifier unit as shown in Figure 7 and Figure 9. This approach not only improves students' understanding of the material, but also enables the students to conduct the experiment effectively in a timely manner. As an introductory-level DC-DC converter lab, the circuits include essential components to prove the basic functionality of converters.

The background theory was covered at the beginning of lab and it was reinforced by simulation and implementation during the lab.

\section{Educational Objectives}

The objectives of the lab are to analyze switching mode power converter topologies and circuits, to utilize BJTs as nonlinear switching devices, to design oscillator circuits, to simulate analog circuits in SPICE, to fabricate the circuits, to measure data, and to compare the results of real 
circuits with that of simulation. The lab component presented here focuses on connecting converter topology theory, SPICE simulation, circuit construction, measurement, and verification.

\section{Laboratory Equipment and Bill of Materials}

Table 1 shows the list of equipment used in the lab. They are all general purpose equipment readily available for undergraduate electrical and computer engineering labs.

Table 1: Laboratory equipment

\begin{tabular}{|l|l|c|l|}
\hline \multicolumn{1}{|c|}{ Description } & \multicolumn{1}{|c|}{ Model } & Qty. & \multicolumn{1}{c|}{ Remark } \\
\hline Oscilloscope & waveJet 322 & 1 & LeCroy, 200 $\mathrm{MHz}$ \\
\hline Function Generator & SFG-2104 & 1 & GW INSTEK, 4 $\mathrm{MHz}$ Synthesized \\
\hline DC Power Supply & E3611A & 1 & HP, for +5 $\mathrm{V}$ input \\
\hline Volt Meter & FLUKE 179 & 2 & FLUKE, Digital Multimeter \\
\hline
\end{tabular}

The lab setup is shown in Figure 1. The DC-DC converter circuits are built on a breadboard and use a single input source of $+5 \mathrm{~V}$ from a DC power supply consuming $20 \mathrm{~mA}$. The circuits convert the $+5 V$ to two voltage sources around $\pm 15 V$ as shown on the voltmeter. The circuits then supply the positive and negative voltages to LM741 op amp circuits, which act as a load attached to the converter. The lab setup would be easily managed by students who have had a prior experience with op amp labs, as the nominal supply voltages of an op amp are typically set to $\pm 15 \mathrm{~V}$.

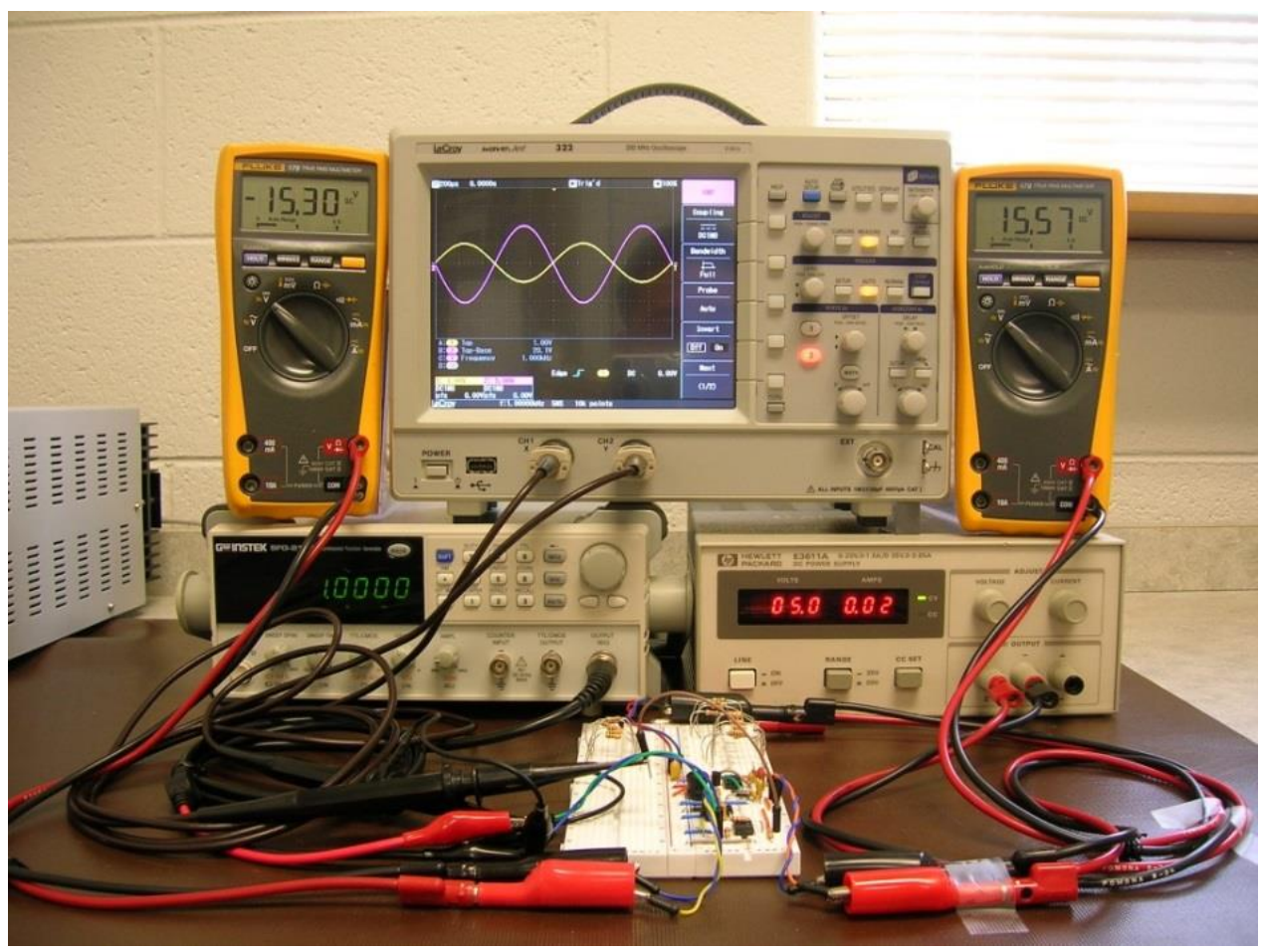

Figure 1: DC-DC converter lab setup 
The main components of the experiment and their bill of materials are listed in Table 2. Note that the transistor leads of the BJT are designated as C-B-E from left to right facing the front of the BJT. An extra caution would be needed when building the circuits as not all compatible $2 \mathrm{~N} 2222 \mathrm{~s}$ are C-B-E. The remaining components were available to students in our lab $(10 k \Omega, 1 k \Omega, 4.7 k \Omega$, $240 \Omega, 0.1 \mu F, 0.01 \mu F$, and $1 n F)$.

Table 2: Main components and bill of materials

\begin{tabular}{|l|l|l|}
\hline \multicolumn{1}{|c|}{ Description } & \multicolumn{1}{c|}{ Digi-key Part Number } & \multicolumn{1}{c|}{ Remark } \\
\hline NE555 Timer IC & 296-1411-5-ND & Max. Frequency: $100 \mathrm{kHz}$ \\
\hline 1 mH Fixed Inductor & M10005-ND & Equiv. Series Resistance: $2.9 \Omega$ \\
\hline 2N2222 NPN BJT & P2N2222AGOS-ND & Leads from left to right: C-B-E \\
\hline LM741 Op Amp & LM741CNNS/NOPB-ND & \\
\hline 1N5819 Schottky Diode & 1N5819GOS-ND & \\
\hline Tantalum $2.2 \mu F / 35 V$ & 478-1871-ND Equivalent & Equiv. Series Resistance $=5.0 \Omega$ \\
\hline
\end{tabular}

\section{Details of Laboratory Experiment}

\section{Lab procedure:}

The following step by step procedures were provided to the students to improve their understanding on the lab and to help them conduct effective experiments.

1) Design an astable multivibrator (oscillator) with a frequency of $100 \mathrm{kHz}$ using the popular timer IC, NE555. (To simplify design and troubleshooting in the lab, the capacitor value of $1 n F$ was given.)

2) Simulate the oscillator circuits and verify through implementation on a breadboard.

3) Add a switching device (BJT) and a collector resistor (e.g. $1 \mathrm{k} \Omega$ ) according to the DC-DC converter topologies to test the switching operation of the BJT. Simulate and verify the circuits.

4) Replace the collector resistor with an energy storage element (inductor). Simulate and verify the circuits.

5) To generate DC voltages, add rectifying and filtering circuits. Simulate and verify the circuits.

6) Measure the voltages on actual circuits and compare to the results of the simulation.

\section{DC-DC Converter Topologies:}

There are mainly three basic types of DC-DC converters; boost, buck and buck-boost. The output voltage of boost converters, also called step-up converters, is always higher than the input voltage. The circuit diagram of the boost converter used in the lab is shown in Figure 2. The NE555 is a versatile timer IC, which is configured for an astable multivibrator operated as a freerunning oscillator. This oscillator provides a switching transistor with a square waveform. 


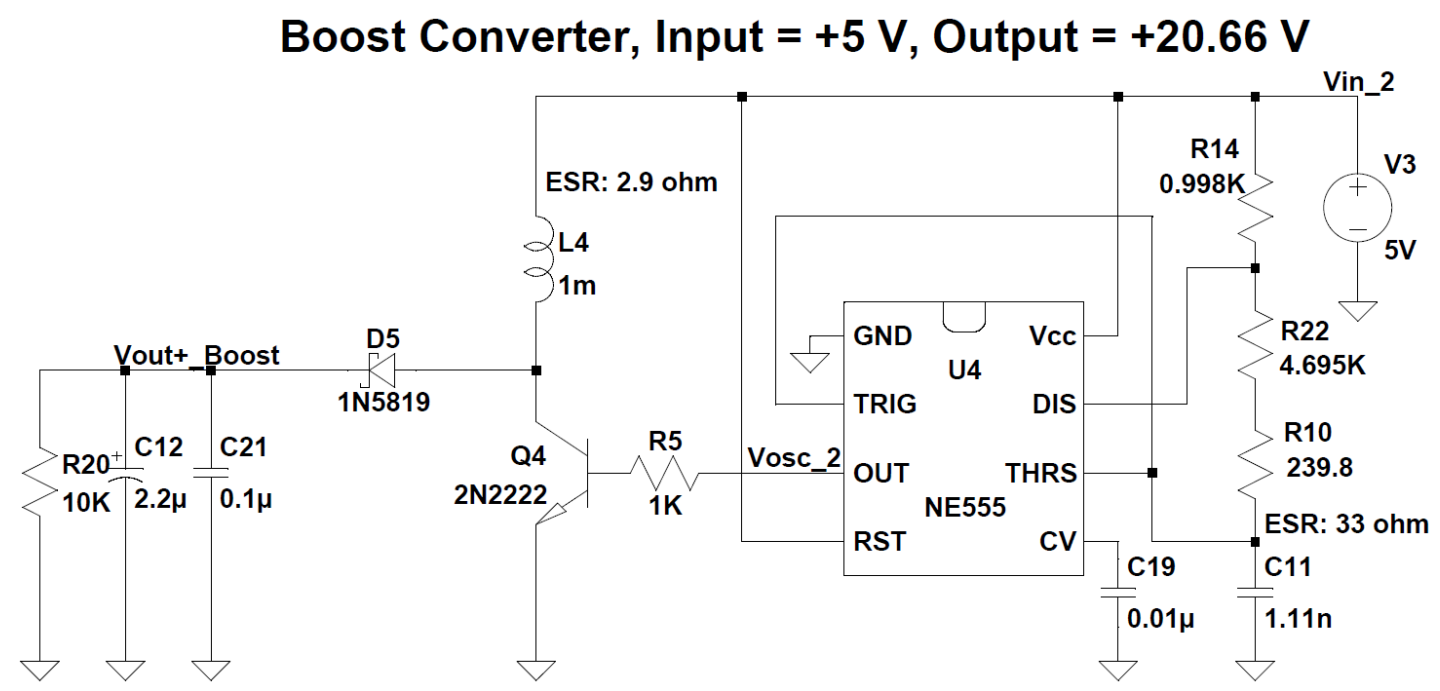

Figure 2: Boost DC-DC converter

The frequency of the NE555 is calculated by the following equation ${ }^{7}$, where $R_{A}=R 14, R_{B}=$ $R 22+R 10$, and $C=C 11$.

$$
\text { Frequency } \approx \frac{1.44}{\left(R_{A}+2 R_{B}\right) C}
$$

The equation can be easily derived by using the $1^{\text {st }}$ order RC circuit's analysis ${ }^{8}$. The lab utilizes LTspice IV simulator ${ }^{9}$, which is free of charge. In simulation the actual values of RC are used for the oscillation as shown in Table 3, to produce as accurate a result as possible. Note that LTspice IV has $0.1 \%$ relative tolerance by default on SPICE.

Table 3: Component values for simulation

\begin{tabular}{|c|c|c|c|l|}
\hline & $\begin{array}{c}\text { Part } \\
\text { Designation }\end{array}$ & $\begin{array}{c}\text { Nominal Values } \\
(\text { Standard 5\%) }\end{array}$ & Actual Values & Instrument used to measure \\
\hline \hline$R_{A}$ & $\mathrm{R} 14$ & $1 \mathrm{k} \Omega$ & $0.998 \mathrm{k} \Omega$ & FLUKE 179, Digital Multimeter \\
\hline$R_{B}$ & $\mathrm{R} 22$ & $4.7 \mathrm{k} \Omega$ & $4.695 \mathrm{k} \Omega$ & FLUKE 179, Digital Multimeter \\
\cline { 2 - 5 } & $\mathrm{R} 10$ & $240 \Omega$ & $239.8 \Omega$ & FLUKE 179, Digital Multimeter \\
\hline$C$ & $\mathrm{C} 11$ & $1 \mathrm{nF}$ & $1.11 \mathrm{nF}$ & $\begin{array}{l}\text { FLUKE PM6304 Programmable } \\
\text { Automatic RCL Meter @ 100 } \\
\text { kHz, ESR: 33 } \Omega\end{array}$ \\
\hline
\end{tabular}

The oscillation frequency of the timer in the simulation is $115.3 \mathrm{kHz}$ as shown in Figure 3 , which is higher than the measurement $(\sim 100 \mathrm{kHz})$ due to the ideal model of the timer IC. The frequency by the above equation gives

$$
\text { Frequency } \approx \frac{1.44}{\left(R_{A}+2 R_{B}\right) C}=\frac{1.44}{(0.998 \mathrm{k} \Omega+2 \times(4.695 \mathrm{k} \Omega+239.8)) \times 1.11 \mathrm{n}}=119.4 \mathrm{kHz}
$$

A bleeder resistor $(R 20,10 \mathrm{k} \Omega)$ is required to keep the output voltage level around $+20 \mathrm{~V}$. If the load is too light (i.e., no load or high resistance), the output voltage would increase higher than 
the maximum voltage rating of the filter capacitors $(C 12 \& C 21)$, which may result in a dielectric breakdown.

Note that the square waveform from the timer goes up to $+5 \mathrm{~V}$ in the simulation, whereas the real signal on the timer output goes up to $+3.6 \mathrm{~V}$, which is close to the typical value of $+3.3 \mathrm{~V}$ as specified in the data sheet ${ }^{7}$. Refer to Figure 10.

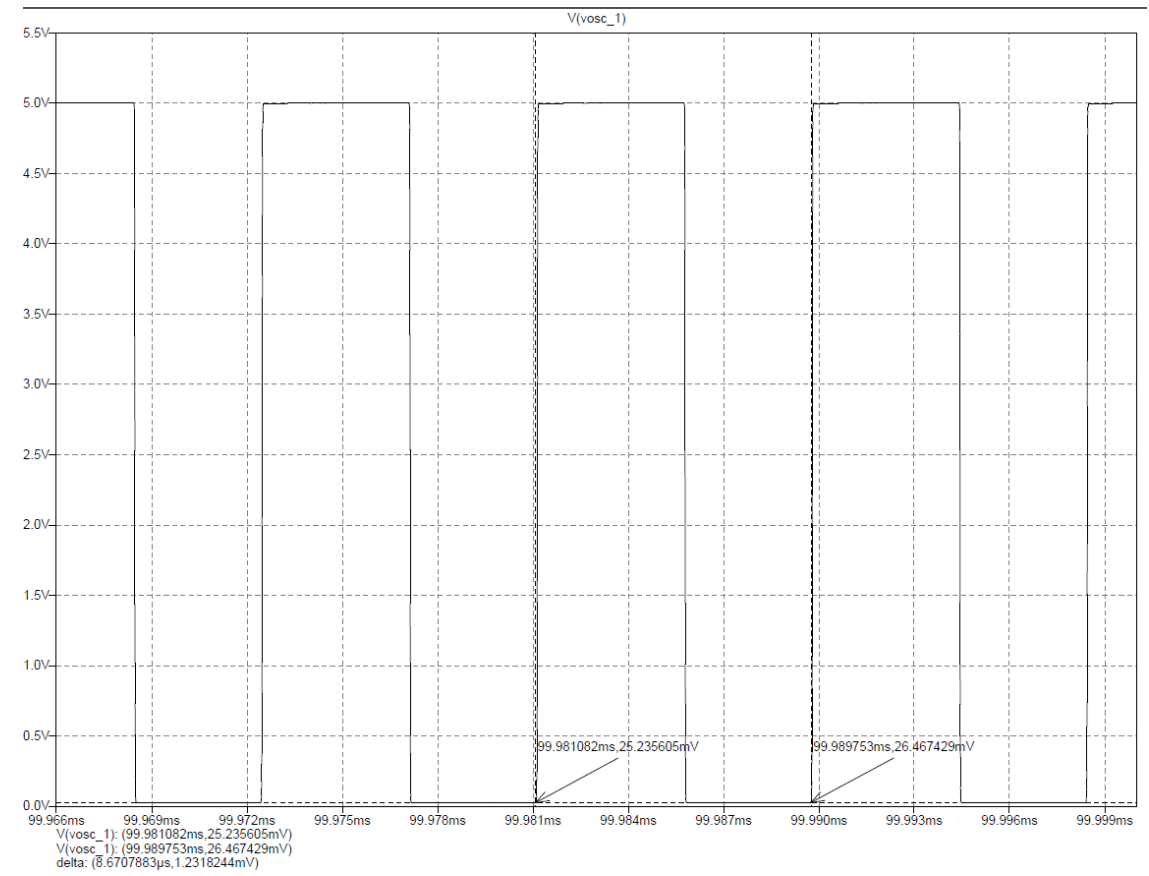

Figure 3: Waveform and frequency of oscillator

For reader's reference the buck and buck-boost types are also shown in Figure 4.
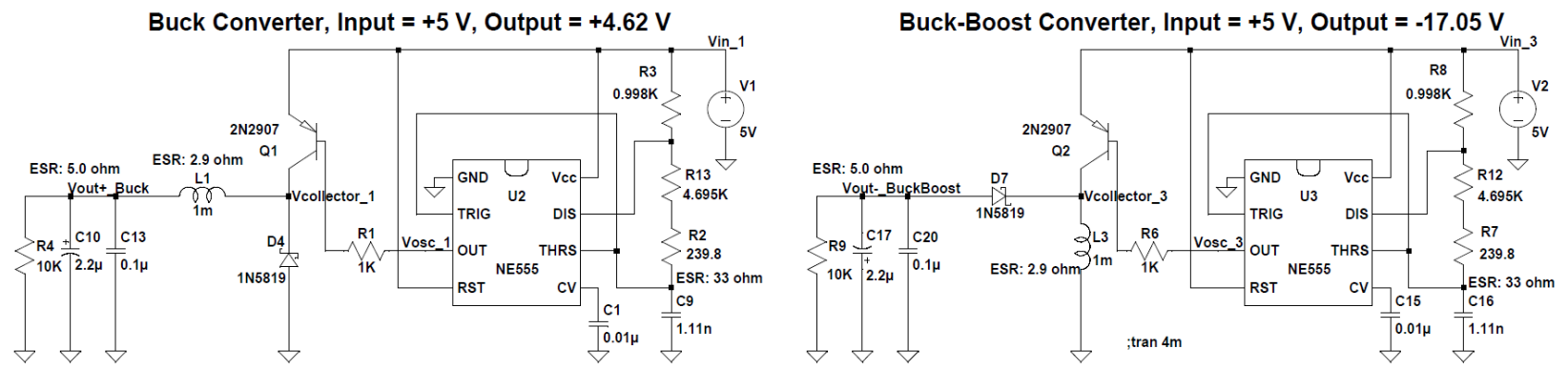

Figure 4: Buck and buck-boost DC-DC converters

The simulation results with proper labels are shown in Figure 5. As shown, the output voltage of boost type $(+22.66 \mathrm{~V})$ is larger than the input voltage $(+5 \mathrm{~V})$, whereas the output of buck type $(+4.62 \mathrm{~V})$ is less than the input. The buck-boost converter has the negative polarity on its output $(-17.05 \mathrm{~V})$. 


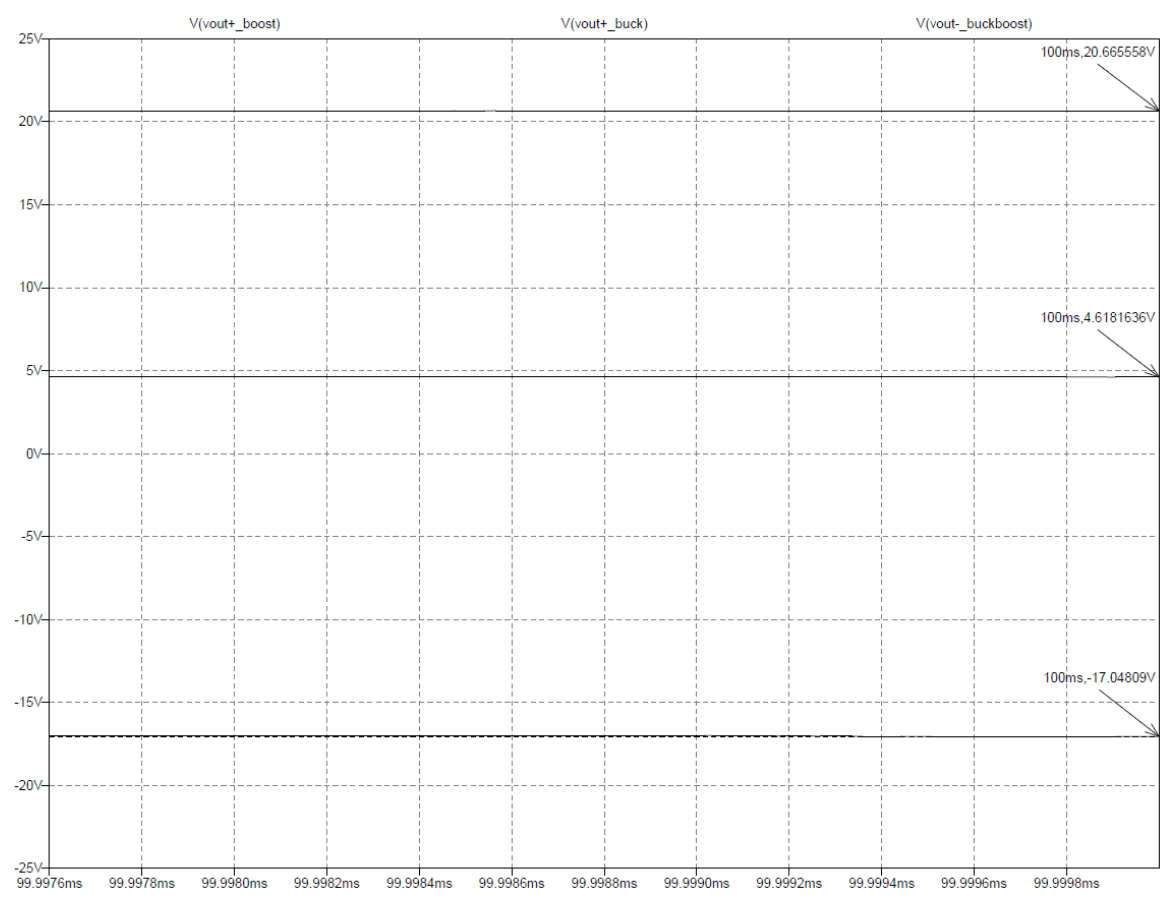

Figure 5: Output Voltages of Boost, Buck and Buck-Boost DC-DC converters

When positive and negative voltage sources are needed for a load, such as an inverting op amp circuits, the boost and buck-booster converters could be utilized together to supply dual voltages. They, however, require two inductors and two BJTs. To make the dual voltage source simpler, a circuit is introduced in Figure 6, which uses one inductor and one BJT. The circuit uses the boost converter with a charge pump circuit to produce a negative voltage. The circuit uses the capacitor $C 5$ as an energy storage element. The simulation shows that the output voltages with $10 \mathrm{k} \Omega$ loads are $-14.43 \mathrm{~V}$ and $+14.64 \mathrm{~V}$.

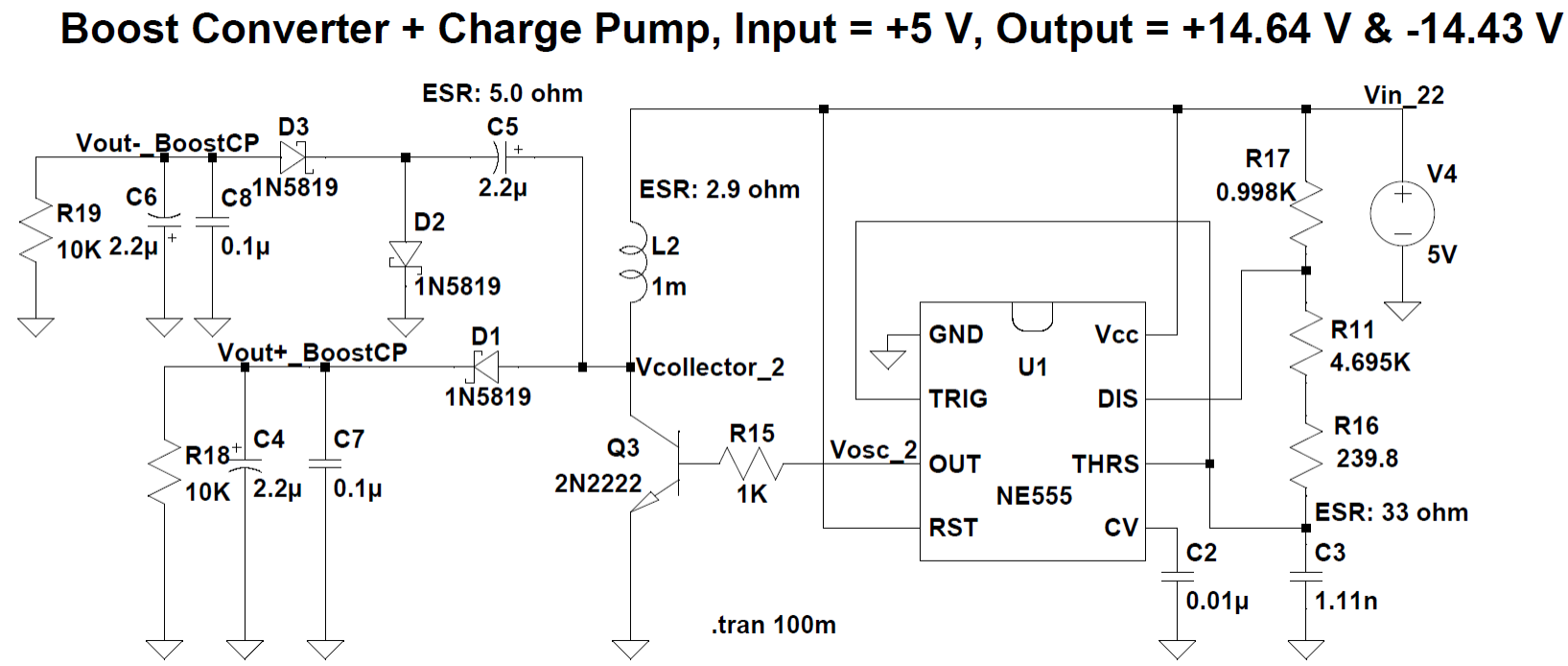

Figure 6: Dual Voltage Supply: Boost and charge pump circuits 
To test the converters with static loads, resistors are attached to the positive and negative voltage source of the real circuits. The ambient temperature was $19.4{ }^{\circ} \mathrm{C}$. The frequency of Vosc_2 ranges from $99.6 \mathrm{kHz} \sim 99.9 \mathrm{kHz}$. The nominal value of the load is set to $10 \mathrm{k} \Omega$ to have the voltage values around $15 \mathrm{~V}$. Table 4 shows \% changes as the load varies. Due to the lack of feedback circuits, it changes $\pm 15 \%$ as the load varies from $7.5 k \Omega$ to $13 k \Omega$.

Table 4: Load regulation from real circuits measured by FLUKE 179

\begin{tabular}{|c|c|c|c|c|}
\hline $\begin{array}{c}\text { Load resistances } \\
(R 18 \text { and } R 19)\end{array}$ & Vout- & Vout+ & $\begin{array}{c}\text { Vout- \% } \\
\text { Change }\end{array}$ & $\begin{array}{c}\text { Vout+ \% } \\
\text { Change }\end{array}$ \\
\hline $7.5 k \Omega$ & $-13.18 V$ & $+13.45 V$ & $15.24 \%$ & $-14.98 \%$ \\
\hline $8.2 k \Omega$ & $-13.91 V$ & $+14.19 V$ & $10.55 \%$ & $-10.30 \%$ \\
\hline $9.1 k \Omega$ & $-14.74 V$ & $+15.02 V$ & $5.21 \%$ & $-5.06 \%$ \\
\hline $10 k \Omega$ & $-15.55 V$ & $+15.82 V$ & $0 \%$ & $0 \%$ \\
\hline $11 k \Omega$ & $-16.37 V$ & $+16.64 V$ & $-5.27 \%$ & $5.18 \%$ \\
\hline $12 k \Omega$ & $-17.09 V$ & $+17.36 V$ & $-9.90 \%$ & $9.73 \%$ \\
\hline $13 k \Omega$ & $-17.82 V$ & $+18.08 V$ & $-14.59 \%$ & $14.29 \%$ \\
\hline
\end{tabular}

To test the converters with dynamic loads and to make use of them practically, an inverting amplifier with a gain of 10 is attached to the dual voltage source as shown in Figure 7 . The amplifier uses the LM741 op amp device model taken from LTspice IV installation (C:IProgram Files (x86)\LTCLLTspiceIVlexamples\Educational\LM741.asc). The absolute maximum ratings of supply voltage of LM741C are $\pm 18 \mathrm{~V}$, therefore the voltage outputs should be kept below the maximum level. This is the reason why the nominal voltage is set to $\pm 15 \mathrm{~V}$.

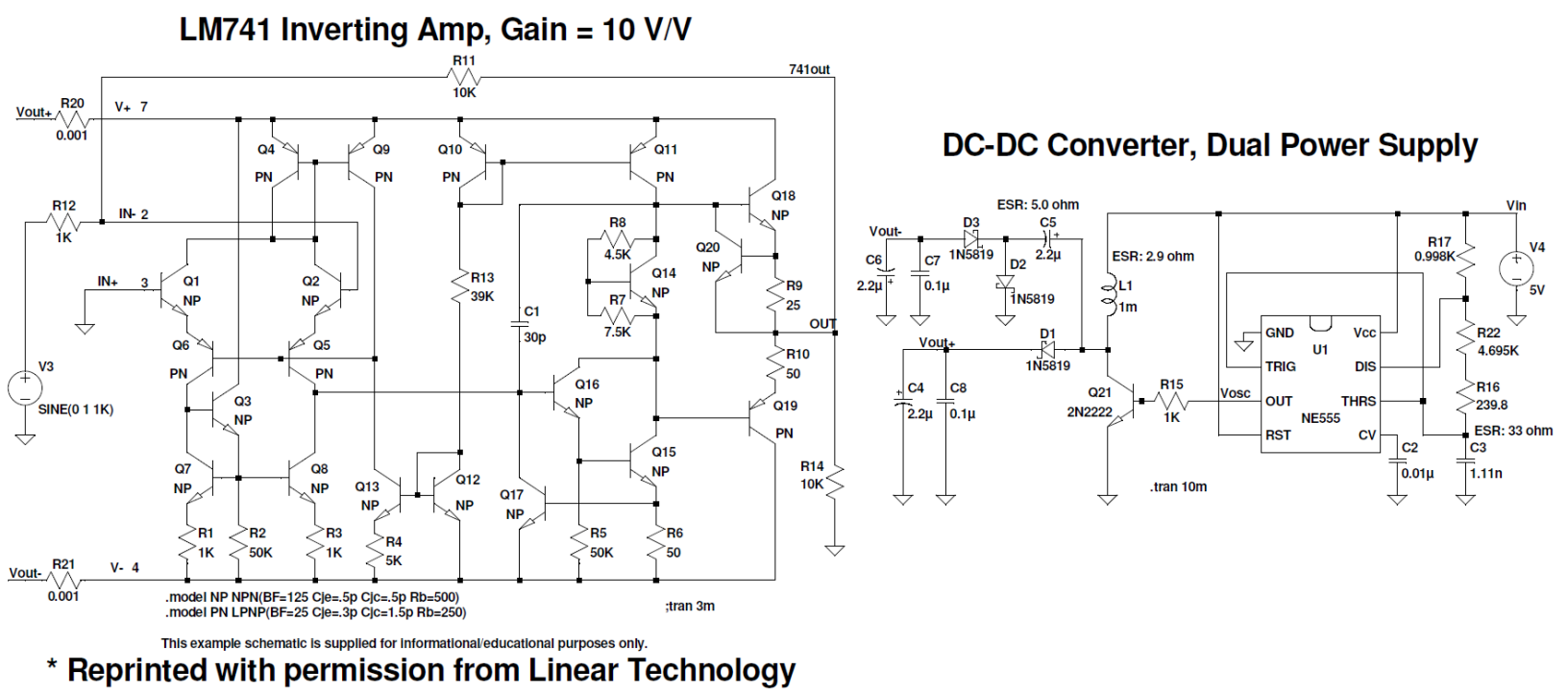

Figure 7: Dual power supply with an inverting amp using 741 op amp

The simulation results are shown in Figure 8. It shows that the output voltage of the converter stays around $\pm 12 \mathrm{~V}$, which are less than the voltages with the nominal load value of $10 \mathrm{k} \Omega$ in Figure $6(+14.64 \mathrm{~V}$ and $-14.43 \mathrm{~V})$ due to the fact that the amp circuit draws more current than 
the resistive loads. The voltages on the actual circuits are $+15.57 \mathrm{~V}$ and $-15.30 \mathrm{~V}$ as shown in Figure 1.

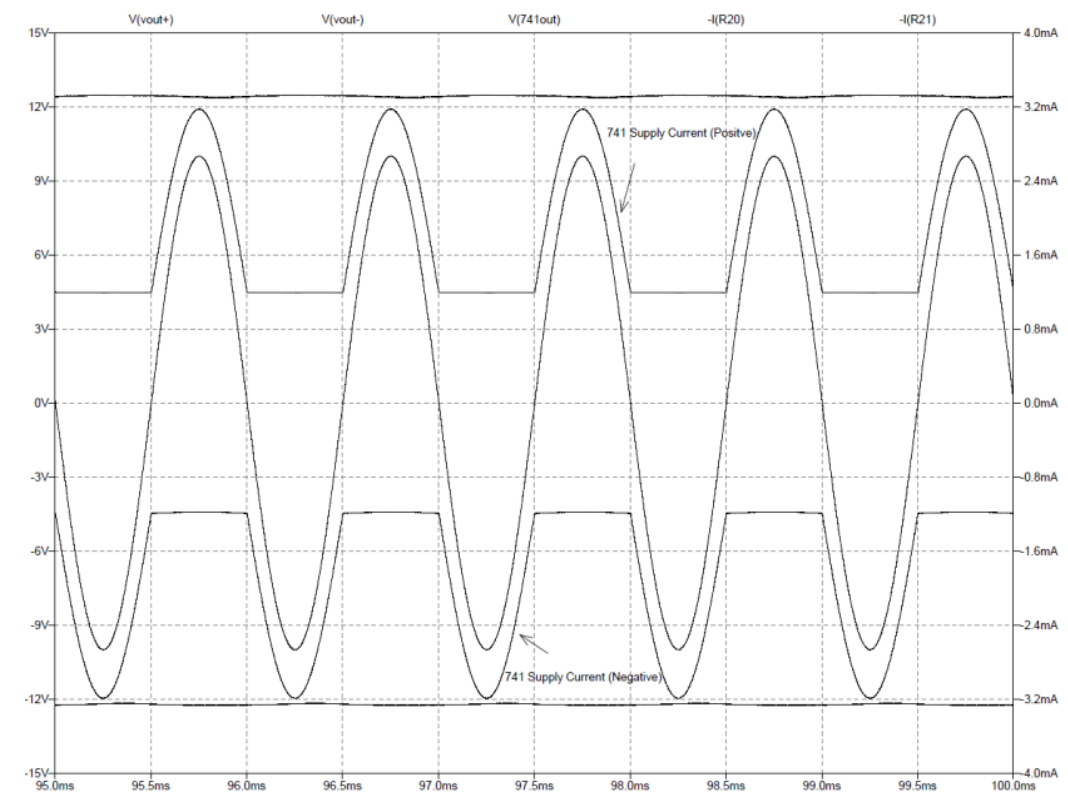

Figure 8: Supply current to 741 op amp and its output waveform

The currents on the amp voltage terminal ranges from $\pm 1.2 \mathrm{~mA}$ to $\pm 3.2 \mathrm{~mA}$, which are more than the currents with the resistive loads $(\sim \pm 1.4 \mathrm{~mA}$ or $\pm 14 \mathrm{~V} / 10 \mathrm{k} \Omega)$. The simulation shows that the inverting amplifier output produces a voltage gain of 10 or $20 \mathrm{~dB}$ when applying an input signal with an amplitude of $1 \mathrm{~V}$.

\section{Component Selections:}

To find the relationship between the inductor values, switching frequency, and output current for a boost converter, the following analysis is used. The boost converter operates either in continuous-conduction mode, where the inductor current is positive, or in discontinuousconduction mode, where the current value can fall to zero. At the boundary of continuousdiscontinuous conduction, the average output current can be found as follows ${ }^{10}$.

$$
I_{O}=\frac{T_{S} V_{O}}{2 L} D(1-D)^{2}
$$

, where $T_{S}$ is the period of switching frequency, $V_{O}$ is the output voltage, $L$ is the inductance, and $D$ is the duty cycle. For the equation above, an assumption is made that the output filter capacitor is very large and the input power is equal to the output power.

For the converter used in this lab, we use the following values to compute the inductance.

1) the output average current is set to $10 \mathrm{~mA} \max$ ( $5 \mathrm{~mA}$ each for positive and negative),

2) $T_{S}$ is equal to $(100 \mathrm{kHz})^{-1}$, 
3) $V_{O}$ is $+15 V$ for a nominal value, and

4) the duty cycle is set to $50 \%$.

By substituting the values in the above equation, the inductance can be computed as follows.

$$
L=\frac{T_{S} V_{O}}{2 I_{O}} D(1-D)^{2}=\frac{\left(10^{-5}\right)(15)}{2\left(10^{-2}\right)} 0.5(1-0.5)^{2}=0.9375 \mathrm{mH}
$$

For a practical inductor value $1 \mathrm{mH}$ is chosen. When the average current is lower than the set value as the lab does, the converter will operate in discontinuous-conduction mode.

As a switching device, the popular TO-92 package of $2 \mathrm{~N} 2222$ is chosen. The maximum collector current of this bipolar transistor is $600 \mathrm{~mA}$. Although this lab uses much less average current than the maximum value, it is used to accommodate a need for future applications easily that would require more power than this lab. It would also provide an opportunity for students to review the materials on the operation of a bipolar NPN transistor. The base current is limited to about $3 m A\left(\frac{V_{O S C}-V_{B E}}{R 15}=\frac{3.6-0.6}{1 K \Omega}\right)$ by the base resistor $R 15$. This current would give the collector current of $225 \mathrm{~mA}$ considering $h_{f e}$ of 75 . The current rating of the inductor is $290 \mathrm{~mA}$, so it would not saturate the inductor.

There will be an inrush current with several times larger than the steady-state input current, which flows through the switching transistor. The transistor should be able to handle this initial stress $^{11}$.

The Schottky diodes are used to accommodate the reduction of forward voltage drops. Note that the negative voltage output in Figure 6 and Figure 7 uses two diodes to have a charge pump operation. Therefore, the negative output suffers from more diode drops than the positive output. As a result, the absolute value of negative voltage is always less than the positive voltage as shown in Table 4.

The more capacitance in the filter, the less ripples on the output. The time to get a steady state output value depends on the filter capacitance. The more capacitance, the longer the simulation time is needed to charge the capacitors. To save the simulation time without compromising the output quality, $2.2 \mathrm{uF}$ capacitors are used for filtering. The larger value can be used to minimize the ripples on the outputs. The ceramic capacitors of $0.1 \mu F$ are used to reduce the spike transient noise caused at the on-off transition.

\section{Circuit layout and measurements:}

The suggested circuit layout on the breadboard is shown in Figure 9. It takes 10 by 30 tie points and two rows as a power bus bar for input $+5 \mathrm{~V}$. The shorter jumper wires are used as much as possible and laid flat on the breadboard to minimize the signal return paths. By doing this, one could minimize the switching noise on the circuits, in which the noise is typically larger than that of linear drop-out power supplies. 
The red/blue pair on top is the input $+5 \mathrm{~V}$ of the circuits feeding from the bench-top HP power supply. The orange/blue pair on the right and the yellow/blue pair on the bottom are the outputs of $+15.57 \mathrm{~V}$ and $-15.30 \mathrm{~V}$, respectively. The input sine wave is connected to the input resistor of the op amp through green/blue pair on the left. All of the pairs are twisted to reduce noise.

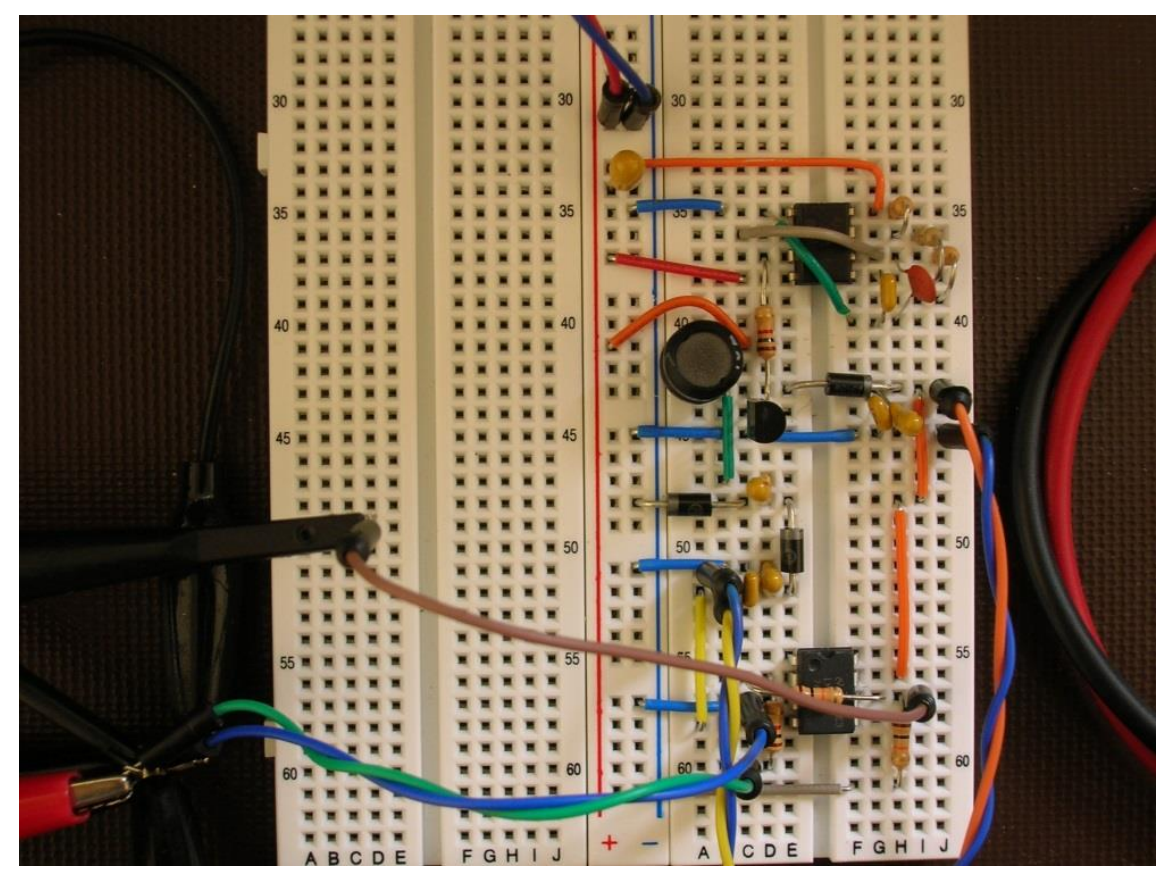

Figure 9: Circuit layout of DC-DC converter with an inverting amp

The output waveform is measured by the oscilloscope and shown in Figure 10. The frequency is $99.92 \mathrm{kHz}$ and the duty cycle is $52.2 \%$.

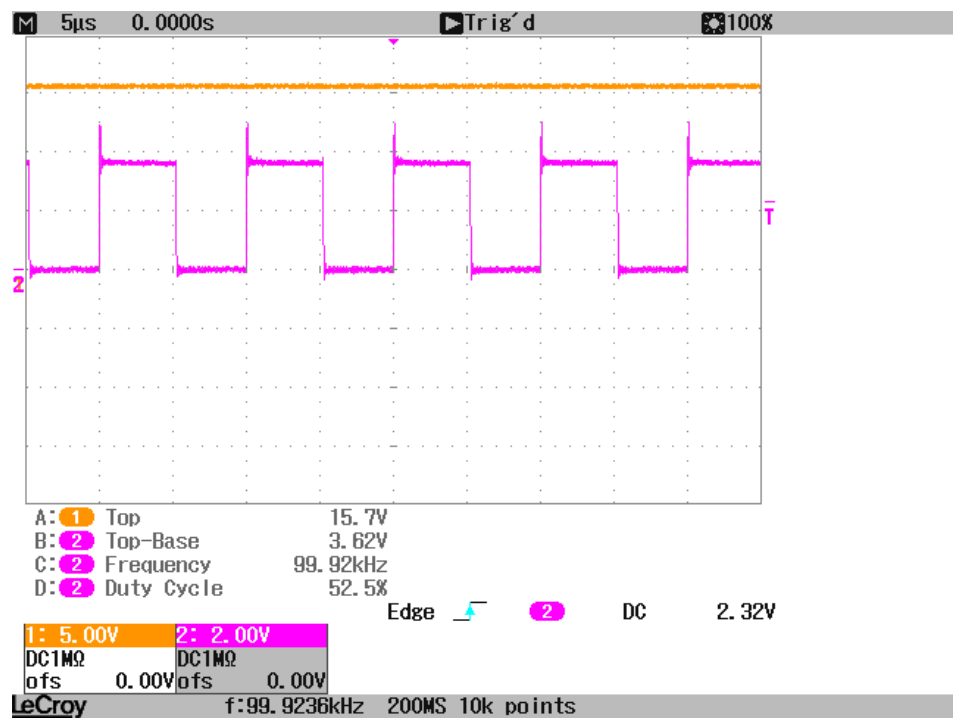

Figure 10: Oscillation frequency and duty cycle of timer IC output 
The input and output waveforms of the inverting amplifier are shown in Figure 11. The amplifier uses the dual voltage outputs generated by the boost converter with the charge pump circuits. It amplifies the input voltage of $1 \mathrm{~V}$ (zero to peak) and generates the output voltage of $10 \mathrm{~V}$ (zero to peak) confirming an inverting amp with a gain of 10 .

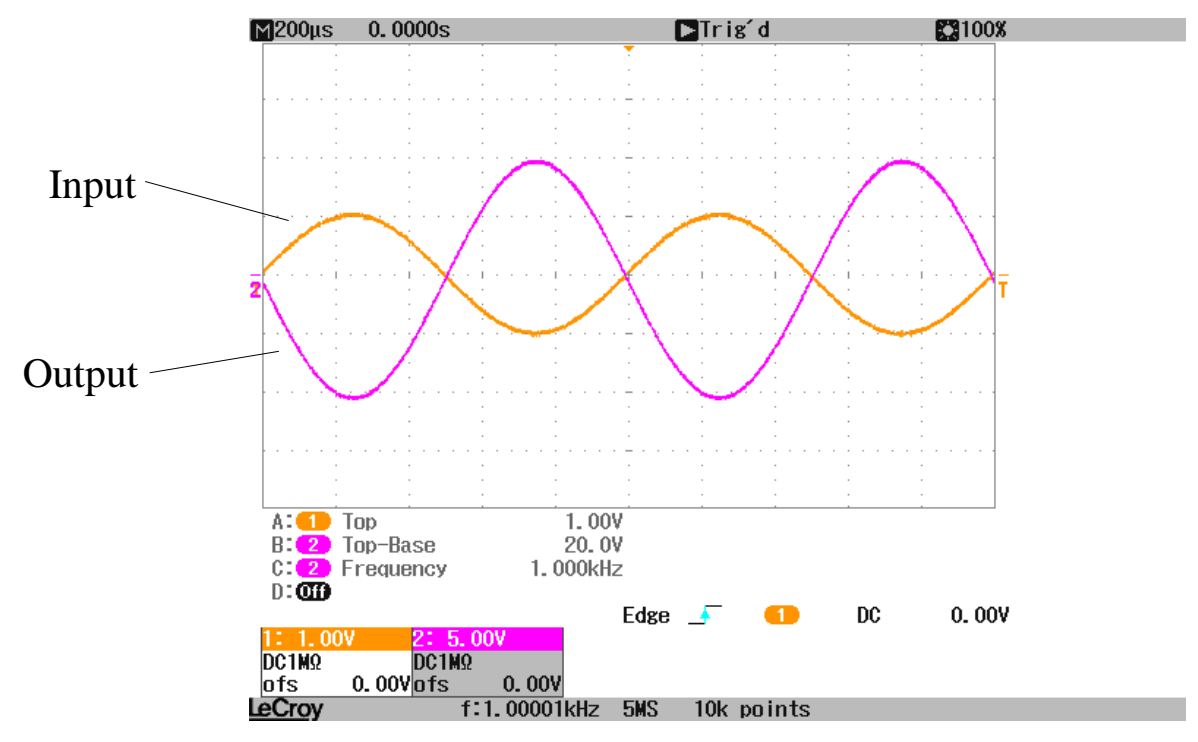

Figure 11: Input and output waveforms of an inverting amplifier

\section{Student Feedback and Assessment Method}

The following survey questions were given to students and the results are shown below each question. A five-point Likert scale was used (1: Strongly Disagree, 2: Disagree, 3: Neutral, 4: Agree, 5: Strongly Agree). The total number of students who completed the survey was 17.

Learning Outcomes: By the end of the lab, students should be able to:

1) Understand that a boost type DC-to-DC converter can produce an output voltage, which is higher than an input voltage.

\begin{tabular}{|c|c|c|c|c|c|c|}
\hline 1: S. D. & 2: D & 3: N & 4: A & 5: S. A. & Avg. & Avg.\% \\
\hline \hline 0 & 0 & 0 & 8 & 8 & 4.5 & 90 \\
\hline
\end{tabular}

2) Comprehend that a DC-DC converter requires an oscillator/astable multivibrator (e.g. 555 Timer IC) to switch a transistor (2N2222) on and off.

\begin{tabular}{|c|c|c|c|c|c|c|}
\hline 1: S. D. & $2: \mathrm{D}$ & $3: \mathrm{N}$ & $4: \mathrm{A}$ & 5: S. A. & Avg. & Avg.\% \\
\hline \hline 0 & 0 & 2 & 10 & 4 & 4.125 & 82.5 \\
\hline
\end{tabular}

3) Understand that a DC-DC converter requires an energy storage element (e.g. inductor or capacitor) to shift the voltage levels.

\begin{tabular}{|c|c|c|c|c|c|c|}
\hline 1: S. D. & 2: D & 3: N & 4: A & 5: S. A. & Avg. & Avg.\% \\
\hline \hline 0 & 0 & 3 & 7 & 6 & 4.1875 & 83.75 \\
\hline
\end{tabular}


4) Analyze DC-DC converter circuits through simulation.

\begin{tabular}{|c|c|c|c|c|c|c|}
\hline 1: S. D. & 2: D & $3: \mathrm{N}$ & 4: A & 5: S. A. & Avg. & Avg.\% \\
\hline \hline 0 & 0 & 4 & 6 & 6 & 4.125 & 82.5 \\
\hline
\end{tabular}

5) Construct the circuits and verify the results by comparing with simulation and by measuring the input and output voltages of the real circuits.

\begin{tabular}{|c|c|c|c|c|c|c|}
\hline 1: S. D. & $2: \mathrm{D}$ & $3: \mathrm{N}$ & $4: \mathrm{A}$ & 5: S. A. & Avg. & Avg.\% \\
\hline 0 & 0 & 1 & 9 & 6 & 4.3125 & 86.25 \\
\hline
\end{tabular}

Overall lab experience:

6) The lab contributed to the understanding of the concept

\begin{tabular}{|c|c|c|c|c|c|c|}
\hline 1: S. D. & 2: D & 3: N & 4: A & 5: S. A. & Avg. & Avg.\% \\
\hline \hline 0 & 0 & 0 & 8 & 8 & 4.5 & 90 \\
\hline
\end{tabular}

7) Students gained a valuable basic knowledge from this lab experience

\begin{tabular}{|c|c|c|c|c|c|c|}
\hline 1: S. D. & 2: D & 3: N & 4: A & 5: S. A. & Avg. & Avg.\% \\
\hline \hline 0 & 0 & 0 & 7 & 9 & 4.5625 & 91.25 \\
\hline
\end{tabular}

\section{Conclusions and Future Work}

Junior students in electrical and computer engineering analyzed, simulated, and fabricated switching mode DC-DC converters successfully in three hours. They measured data on real circuits and compared the results of real circuits with that of LTspice IV simulation. The comparison between the simulation and the implementation indicated good agreement.

The approach was taken to integrate students' prior knowledge and experiences on BJTs, diodes, inductors, and capacitors. The step by step procedures were used to conduct the experiments effectively and to improve students' understanding.

The lab experiment used basic equipment available in undergraduate electrical and computer engineering labs and the circuits used general purpose electronic components available in the labs. This paper includes lab experiments materials in detail, including complete working schematics, bill of materials, photos, simulation results, and oscilloscope screens.

Based on the survey results in Section 5, students appreciated the lab experiment. It was indicated that the lab experiment presented in this paper helped students understand the concept and gain knowledge about switching mode DC-DC converters.

Future work will involve adding additional circuits for regulating the output voltages under the different load conditions by PWM control circuits and developing an experiment that can design and test a DC-DC converter with a higher output power range. 


\section{References}

1. NASA, "SM2A-03-BLOCK II-(1) Apollo Operations Handbook (2.6 Electrical Power System)," http://www.ibiblio.org/apollo/ApolloProjectOnline/Documents/SMA2A-03BLOCK\%20II\%20Volume\%201\%2019691015/aoh-v1-2-06-eps.pdf

2. A. Rubaai, "Teaching Power Electronics Converter Experiments that Integrates Fuzzy Logic Approach," Proc. 2011 ASEE Annual Conference and Exposition, 2011

3. R. Belu, "A Project-based Power Electronics Course with an Increased Content of Renewable-energy Applications", Proc. 2009 ASEE Annual Conference and Exposition, 2009

4. W. Thain, "A Laboratory Component of a Switching Power Supply Course Requiring Nominal Resources," Proc. 2009 ASEE Southeast Section Conference, 2009

5. P. Idowu, "Development of Simulation Models for Power Converters - Undergraduate Research Experience," Proc. 2005 ASEE Annual Conference and Exposition, 2005

6. D. S. Zinger, “An Introductory Power Electronics Course Laboratory,” Proc. 1999 ASEE Conference, 1999

7. Texas Instruments, NE555P, http://www.ti.com/lit/ds/symlink/ne555.pdf

8. A. S. Sedra and K. C. Smith, "Microelectronics Circuits (Chap 17.7)," 6th ed., Oxford University Press, ISBN13: 978-0-19-532303-0, 2010

9. Linear Technology, LTspice IV Simulator, http://www.linear.com/designtools/software/\#LTspice

10. N. Mohan, T. M. Undeland, and W. P. Robbins, "Power Electronics: Converters, Applications, and Design (Chap 7-4),” 2nd ed., John Wiley \& Sons Inc., ISBN: 0-471-58408-8, 1995

11. S. S. Ang, "Power-Switching Converters (Chap 2)," 1st ed., Marcel Dekker Inc., ISBN: 0-8247-9630-6, 1995 